\title{
El profesorado de Educación Física ante la situación de educación a distancia por Covid-19
}

\author{
Physical Education teachers facing the situation of distance education due to Covid-19 \\ Teresa Fuentes Nieto ${ }^{1}$, Borja Jiménez Herranz ${ }^{2}$, Víctor Manuel López-Pastor ${ }^{1}$, \\ Carla Fernández-Garcimartín ${ }^{\mathbf{1}}$
}

1 Facultad de Educación de Segovia. Universidad de Valladolid, España.

2 Maestro de Educación Física. CRA El Pinar, Navas de Oro, Segovia, España.

CORRESPONDENCIA:

Teresa Fuentes Nieto

fuentes.teresa@gmail.com

Recepción: octubre 2020 • Aceptación: enero 2021
CÓMO CITAR EL ARTÍCULO:

Fuentes, T., Jiménez, B., López-Pastor, V. M. \& FernándezGarcimartín, C. (2021). El profesorado de Educación Física ante la situación de educación a distancia por Covid-19. Cultura, Ciencia y Deporte, 16(49), 505-517. http://dx.doi.org/10.12800/ ccd.v16i49.1589

\section{Resumen}

Este estudio pretende analizar la percepción del profesorado de Educación Física (EF) sobre diferentes aspectos de la educación a distancia en España como consecuencia de la pandemia Covid-19. Se ha aplicado un cuestionario on-line diseñado ad hoc a 526 docentes de EF. Los resultados muestran diferencias significativas entre un primer periodo (13 de marzo-4 de abril) y un segundo periodo (15 de abril-final de curso). Los centros educativos se han adaptado a las nuevas circunstancias de forma positiva y los docentes se han sentido cada vez menos inseguros. La mayoría del profesorado ha utilizado una evaluación formativa. A la hora de calificar, la mayor parte se ha aproximado a lo que marca la legislación educativa (Orden EFP/365/2020), sin cumplirla exactamente, dado que no han utilizado la tercera evaluación de forma positiva para los estudiantes que sí han estado conectados. Por último, consideran que la EF debería tener un rol más importante durante el confinamiento que el que perciben que ha tenido. Parece necesario realizar más investigación sobre cómo el profesorado de EF ha abordado esta etapa tan extraña, así como generar dinámicas de formación permanente para mejorar la labor docente durante el curso 2020-21.

Palabras clave: confinamiento, coronavirus, enseñanza on-line, evaluación, percepción del profesorado.

\section{Abstract}

This study aims to analyse the perception of Physical Education (PE) teachers on different aspects of on-line education in Spain due to the Covid-19 pandemic. An on-line questionnaire designed "ad hoc" has been applied to $526 \mathrm{PE}$ teachers. The results show significant differences between a first period (March 13th-April 4th) and a second period (April 15th-end of course). Educational centres have adapted to the new circumstances in a positive way and teachers have felt less and less insecure. Most of the teachers have used a formative assessment. When grading, most of the teachers have approached what the educational legislation indicates (Order EFP / 365/2020), without fulfilling it exactly, because they have not used the third evaluation in a positive way for students who have been connected. Finally, it considers that the PE should have a more important role during confinement than the one they perceive they have had. It seems necessary to carry out more research about how PE teachers have approached this strange period, as well as to generate dynamics of permanent training to improve teaching work during the 2020-21 academic year.

Key words: lockdown, coronavirus, on-line teaching, assessment, teacher's perception. 


\section{Introducción}

Desde la llegada de la pandemia Covid-19, nuestro estilo de vida se ha visto modificado, afectando al ámbito educativo de manera importante. La extensión de la Covid-19 en España hizo que, durante la segunda semana de marzo de 2020, el Gobierno decidiera cerrar los centros educativos, confinar en sus hogares a toda la población y que todo el sistema educativo se desarrollara de forma telemática, renovándose su regulación continuamente. Por tanto, el profesorado de Educación Física (EF) de Primaria y Secundaria se ha encontrado con una situación absolutamente imprevista y que ha alterado considerablemente la docencia y la programación.

La evolución de la pandemia obligó a las instituciones a publicar diferentes textos legislativos y actuaciones educativas. En la Tabla 1 se muestra un resumen cronológico de las principales actuaciones a nivel estatal y autonómico llevadas a cabo en España.

Como se ha visto, la pandemia Covid-19 ha generado que todo el sistema educativo español haya pasado de una enseñanza presencial a una enseñanza on-line de una forma completamente imprevista e inmediata. Los centros escolares, el profesorado y las familias se han tenido que adaptar al marco legislativo implementado durante este periodo.

La situación ha dejado al profesorado sorprendido, en una postura complicada, incluido el de EF (Martínez-Egea, 2020). En Fuentes et al. (2020) puede encontrarse un estudio cualitativo sobre la vivencia de un grupo de profesores de EF durante los tres primeros meses del confinamiento. Los resultados muestran que vivieron una sensación de agobio generalizado durante las primeras tres semanas (marzo de 2020), donde abundan los sentimientos de incertidumbre e inseguridad, y que esta visión cambió tras las vacaciones de Semana Santa (segunda semana de abril), donde la mayoría de los maestros de EF pudieron organizarse mejor, comenzar a planificar qué hacer durante el tercer trimestre e ir adaptándose a esta nueva situación.

Por otra parte, la Orden EFP/365/2020 estableció flexibilizar y adaptar las programaciones, dando prioridad a los contenidos esenciales. Parece que los especialistas de EF de los diferentes niveles educativos se ocuparon, en un primer momento, de proponer actividades que garantizaran un mínimo de actividad física corporal entre el alumnado confinado, siguiendo las recomendaciones de la Organización Mundial de la Salud (OMS) sobre la importancia de que los niños y adolescentes entre 10 y 17 años realicen al menos 60 minutos al día de actividad física entre moderada y vigorosa (OMS, 2010). En una segunda fase, en la que se produjo un intercambio de recursos y experiencias entre el profesorado del área de EF, se propusieron actividades en forma de desafíos que propiciaran la recuperación de actividades más lúdicas y atractivas para el alumnado, las cuales, a su vez, proporcionaran momentos de esparcimiento y ocio activo desde el confinamiento de sus hogares (Martínez-Egea, 2020). En este mismo sentido, Fuentes et al. (2020) encontraron que muchos maestros de EF habían introducido conte-

Tabla 1. Cronología sobre algunas actuaciones realizadas a nivel estatal y autonómico.

\begin{tabular}{|c|c|c|}
\hline Fecha & Organismo & Actuaciones \\
\hline $\begin{array}{l}\text { 10-14 de marzo } \\
\text { de } 2020\end{array}$ & $\begin{array}{l}\text { Consejerías de } \\
\text { Educación }\end{array}$ & Suspensión de toda actividad docente presencial y pasar a educación on-line. \\
\hline $\begin{array}{l}14 \text { de marzo de } \\
2020\end{array}$ & Consejo de Ministros & $\begin{array}{l}\text { Publicación del Real Decreto 463/2020. Declaración del Estado de Alarma para la gestión de } \\
\text { la crisis sanitaria ocasionada por la Covid-19. }\end{array}$ \\
\hline $\begin{array}{l}\text { 16-31 de marzo } \\
\text { de } 2020\end{array}$ & $\begin{array}{l}\text { Comunidades } \\
\text { Autónomas }\end{array}$ & $\begin{array}{l}\text { Publicación de diferentes órdenes e instrucciones sobre el funcionamiento de los centros: } \\
\text { - Teletrabajo y videoconferencias. } \\
\text { - Flexibilidad horaria. } \\
\text { - Turnos escalonados. } \\
\text { - Evaluación del segundo trimestre (valoración de lo trabajado hasta el momento de forma } \\
\text { presencial y de forma telemática si lo hubiera). }\end{array}$ \\
\hline $\begin{array}{l}15 \text { de abril de } \\
2020\end{array}$ & $\begin{array}{l}\text { Ministerio de } \\
\text { Educación y Formación } \\
\text { Profesional }\end{array}$ & $\begin{array}{l}\text { Publicación de la Orden EFP/365/2020. Principales directrices a tener en cuenta en el tercer } \\
\text { trimestre: } \\
\text { a- Cuidar a las personas, un principio fundamental. } \\
\text { b- Mantener la duración del curso escolar. } \\
\text { c- Adaptar la actividad lectiva a las circunstancias. } \\
\text { d- Flexibilizar el currículo y las programaciones didácticas. } \\
\text { e- Adaptar la evaluación, promoción y titulación: evaluación continua, prioridad a los } \\
\text { contenidos no trabajados y adquisición de aprendizajes mínimos. } \\
\text { f- Trabajar de manera coordinada (instituciones, centros y profesorado). } \\
\text { g- Preparar el próximo curso } 2020 / 21 \text { : informes individualizados con los aprendizajes no } \\
\text { alcanzados en el curso } 2019 / 20 \text { y plan de refuerzo para el curso } 2020 / 21 \text {. }\end{array}$ \\
\hline
\end{tabular}


nidos variados durante el confinamiento, priorizando actividades lúdicas y retos motrices que se podían realizar en casa de manera individual o en familia, manteniendo activos a los estudiantes para que les ayudase a liberar la posible tensión psicológica por la situación.

Martínez-Hita (2020) llevó a cabo un estudio sobre los intereses y motivaciones del alumnado y las propuestas utilizadas en el área de EF durante el periodo de confinamiento por Covid-19. Destaca que las propuestas de contenido de EF mejor valoradas por los estudiantes fueron: juegos y actividades deportivas en casa, entrenamientos con apoyo musical y retos motrices. Asimismo, Shahidi et al. (2020) señalan las actividades circenses (acrobacias, manipulaciones, equilibrios, etc.) como un contenido popular en las clases de EF e idóneo para el periodo de confinamiento. Por su parte, en el estudio desarrollado por Baena-Morales et al. (2021) la condición física y salud (ejercicios con autocargas, subir y bajar escaleras, etc.) resulta ser el contenido más trabajado durante el primer mes de educación a distancia según el profesorado de EF encuestado.

Desde el año 2014, España tiene un currículum oficial de EF que organiza sus contenidos según la propuesta denominada "Programar por Dominios de Acción Motriz” (Julián et al., 2016; Larranz, 2004; López-Pastor et al., 2016). Los Decretos oficiales que lo desarrollan son: el RD 126/2014 para Educación Primaria y el RD 1105/2014 para Educación Secundaria. En la Tabla 2 presentamos un resumen de los bloques de contenidos en que está organizado el currículum de EF en cada etapa.

La evaluación es uno de los aspectos clave en todo proceso educativo (Blázquez, 2017; López-Pastor, 2006; Sanmartín, 2007). No obstante, las circunstancias han hecho que este proceso sea sometido a ciertos cambios o ajustes para que siga manteniendo su esencia formativa. En lo relativo a la evaluación, la Orden EFP/365/2020 destaca: (a) la importancia de adaptar el proceso de evaluación con el objetivo prioritario de que los estudiantes no pierdan el curso y puedan continuar avanzando en su formación; (b) tener en cuenta las circunstancias de los estudiantes más vulnerables; (c) dar prioridad a una for- ma de evaluación de los aprendizajes que sea continua y tenga un carácter diagnóstico y formativo; (d) utilizarla para planificar medidas de refuerzo y programar para el siguiente curso escolar. En lo relativo a la calificación pide que se dé prioridad a los dos primeros trimestres, y que el estudiante no se vea perjudicado por la situación generada en el tercer trimestre.

Un análisis didáctico de esta orden muestra que da más importancia a los procesos de evaluación formativa y continua que a la evaluación sumativa y final, aunque no diferencia claramente entre evaluación y calificación. Cada vez hay más autores dentro de la EF que defienden estos mismos planteamientos (Herrero-González et al., 2020; Blázquez, 2017; López-Pastor, 2006; López-Pastor y Pérez-Pueyo, 2017; Lorente-Catalán, 2008). En un estudio reciente, Fuentes et al. (2020) comprueban que el profesorado de EF que venía realizando habitualmente procesos de evaluación formativa los ha seguido utilizando durante el confinamiento, adaptando los instrumentos y procedimientos a la nueva situación y legislación.

Se puede afirmar que tanto esta base teórica, comola legislación y la aplicación práctica de algunos profesores de EF, está en sintonía con la declaración oficial de la Asociación Internacional de Escuelas Superiores de EF (AIESEP) sobre la evaluación en EF (AIESEP, 2020). Esta declaración pone el énfasis, entre otras cosas, en que el sentido de la "evaluación para el aprendizaje" y la "evaluación del aprendizaje" tienen diferentes propósitos y no se excluyen mutuamente. Sin embargo, la EF siempre debería incluir aspectos de evaluación para el aprendizaje.

Por último, parece relevante señalar las diferentes posiciones en cuanto a la importancia de la EF en esta situación tan excepcional. La pandemia del Covid-19 ha restringido la actividad física en personas de todas las edades, por lo que es muy importante que las familias y educadores no permitan que los niños adopten estilos de vida más sedentarios y que aumenten el tiempo de pantalla (Shahidi et al., 2020). Por tanto, una de las prioridades del profesorado de EF durante esta pandemia sería lograr que su alumnado realizase

Tabla 2. Contenidos de EF en España según la etapa educativa (RD 126/2014 y 1105/2014).

\begin{tabular}{ll}
\hline Educación Primaria & Educación Secundaria \\
\hline 1- Acciones motrices individuales en entornos estables. & 1- Acciones motrices individuales en entornos estables. \\
\hline 2- Acciones motrices en situaciones de oposición. & 2- Acciones motrices en situaciones de oposición. \\
\hline $\begin{array}{l}\text { 3- Acciones motrices en situaciones de cooperación, con o sin } \\
\text { oposición. }\end{array}$ & $\begin{array}{c}\text { 3- Acciones motrices en situaciones de cooperación, con o sin } \\
\text { oposición. }\end{array}$ \\
\hline $\begin{array}{l}\text { 4- Acciones motrices en situaciones de adaptación al entorno físico. } \\
\begin{array}{l}\text { 5- Acciones motrices en situaciones de índole artística o de } \\
\text { expresión. }\end{array}\end{array}$ & $\begin{array}{l}\text { 5-Acciones motrices en situaciones de adaptación al entorno. } \\
\text { expresión. }\end{array}$ \\
\hline
\end{tabular}


actividad física de forma diaria, como forma de salud y de superar la situación de confinamiento. En este sentido, Silva-Filho et al. (2020) señalan que el profesor de EF debe ser considerado un profesional de la salud esencial durante este período, porque puede orientar y estimular a las personas para que practiquen ejercicio físico de manera habitual. Martínez-Egea (2020) coincide en esta idea e indica que no puede olvidarse el peso de esta materia en el currículum educativo, y mucho menos ante crisis sanitarias como la que padecemos, en las que más que nunca se hace necesario la actividad física en las rutinas del alumnado para la mejora del bienestar físico y mental. Fuentes et al. (2020) encuentran que un grupo de maestros de EF considera que se trata de un área fundamental en estas situaciones, pero que también se debe pensar muy bien qué actividades y tareas realizar en EF y la carga de trabajo que está recibiendo el alumnado (y sus familias) semana a semana.

Aunque en estos pocos meses se han publicado algunos estudios con reflexiones y propuestas de docentes o grupos de docentes de EF, así como trabajos con muestras pequeñas, no se han encontrado investigaciones sobre la temática con grandes muestras que permitan tener una visión más amplia de cómo ha vivido el profesorado de EF esta situación de confinamiento por la pandemia Covid-19. En ese sentido, este trabajo pretende analizar cómo ha afectado esta situación a los docentes de EF españoles, cómo han actuado durante los meses de enseñanza a distancia y qué decisiones y soluciones han ido encontrando para desarrollar su labor docente.

De una forma más detallada, los objetivos del estudio son los siguientes:

1. Comprobar si existen diferencias entre antes y después de las vacaciones de Semana Santa (entre el 3 y el 13 de abril de 2020) en las siguientes variables: (a) organización de los centros y coordinación entre el profesorado; (b) contenidos de EF trabajados; (c) sensaciones y vivencias del profesorado de EF ante la situación.

2. Analizar qué tipo de sistemas y procesos de evaluación ha utilizado el profesorado de EF durante estos meses.

3. Conocer el pensamiento del profesorado de EF sobre el papel del área durante la situación de confinamiento por la Covid-19.

\section{Metodología}

Se ha llevado a cabo un estudio exploratorio, basado en la aplicación de un cuestionario on-line a una muestra elevada, formada por 526 docentes de EF de Primaria y Secundaria de toda España. Sus características principales se presentan en las Tablas 3 y 4.
Tabla 3. Datos descriptivos de la muestra.

\begin{tabular}{|c|c|c|c|}
\hline $\begin{array}{l}\text { Variables } \\
\text { Descriptivas } \\
(\mathrm{N}=526)\end{array}$ & Opciones & Sujetos & Porcentaje \\
\hline \multirow[t]{2}{*}{ Sexo } & Mujer & 240 & 45.6 \\
\hline & Hombre & 286 & 54.4 \\
\hline \multirow[t]{5}{*}{ Edad } & $20-30$ & 51 & 9.7 \\
\hline & $31-40$ & 190 & 36.1 \\
\hline & $41-50$ & 202 & 38.4 \\
\hline & $51-60$ & 78 & 14.8 \\
\hline & 61 o más & 5 & 1.0 \\
\hline \multirow[t]{2}{*}{ Entorno } & Rural & 167 & 31.7 \\
\hline & Urbano & 359 & 68.3 \\
\hline \multirow{5}{*}{$\begin{array}{l}\text { Nivel } \\
\text { sociocultural del } \\
\text { alumnado }\end{array}$} & Bajo & 26 & 4.9 \\
\hline & Medio-Bajo & 134 & 25.5 \\
\hline & Medio & 256 & 48.7 \\
\hline & Medio-Alto & 101 & 19.2 \\
\hline & Alto & 9 & 1.7 \\
\hline \multirow[t]{3}{*}{ Titularidad } & Pública & 424 & 80.6 \\
\hline & Concertada & 85 & 3.2 \\
\hline & Privada & 17 & 16.2 \\
\hline \multirow{3}{*}{$\begin{array}{l}\text { Nivel educativo } \\
\text { en que trabajan }\end{array}$} & Ed. Primaria & 276 & 52.5 \\
\hline & ESO & 213 & 40.5 \\
\hline & Primaria y ESO & 37 & 7.0 \\
\hline
\end{tabular}

Tabla 4. Distribución de la muestra por comunidades autónomas.

\begin{tabular}{|c|c|c|}
\hline & Sujetos & Porcentaje \\
\hline Andalucía & 52 & 9.9 \\
\hline Aragón & 51 & 9.7 \\
\hline Asturias & 28 & 5.3 \\
\hline Cantabria & 12 & 2.3 \\
\hline Castilla-La Mancha & 18 & 3.4 \\
\hline Castilla y León & 97 & 18.4 \\
\hline Cataluña & 28 & 5.3 \\
\hline Extremadura & 14 & 2.7 \\
\hline Galicia & 13 & 2.5 \\
\hline Islas Baleares & 8 & 1.5 \\
\hline Canarias & 18 & 3.4 \\
\hline La Rioja & 44 & 8.4 \\
\hline Madrid & 60 & 11.4 \\
\hline Murcia & 33 & 6.3 \\
\hline Navarra & 5 & 1.0 \\
\hline País Vasco & 14 & 2.7 \\
\hline Valencia & 25 & 4.8 \\
\hline Ceuta & 2 & 0.4 \\
\hline Melilla & 4 & 0.8 \\
\hline
\end{tabular}


Tabla 5. Dimensiones, ítems y variables que abordan los objetivos de nuestro estudio.

\begin{tabular}{|c|c|c|c|}
\hline Dimensión & Ítem & Variable & Escala \\
\hline \multirow{7}{*}{$\begin{array}{l}\text { 1. Diferencias } \\
\text { entre las } \\
\text { dos etapas } \\
\text { temporales. }\end{array}$} & 11 & $\begin{array}{l}\text { Antes de Semana Santa: ¿cómo consideras que fue la respuesta del centro/ } \\
\text { equipo directivo ante la situación creada con el confinamiento de escolares (a } \\
\text { nivel de coordinación entre profesores, etc.)? }\end{array}$ & Likert, 5 opciones \\
\hline & 12 & $\begin{array}{l}\text { Después de Semana Santa: ¿cómo consideras que ha sido la respuesta del } \\
\text { centro/equipo directivo ante la situación creada con el confinamiento de } \\
\text { escolares (a nivel de coordinación entre profesores, etc.)? }\end{array}$ & Likert, 5 opciones \\
\hline & 13 & $\begin{array}{l}\text { ¿En qué grado has tenido que variar los contenidos de tu programación debido } \\
\text { a la situación de confinamiento por el Covid19? }\end{array}$ & Likert, 4 opciones \\
\hline & 14 & $\begin{array}{l}\text { Durante las tres primeras semanas de confinamiento (en marzo, antes de } \\
\text { Semana Santa) ¿qué contenidos de EF has trabajado? }\end{array}$ & 5 opciones \\
\hline & 15 & $\begin{array}{l}\text { Después de Semana Santa (abril, mayo y junio): ¿Qué contenidos de EF has } \\
\text { trabajado? }\end{array}$ & 5 opciones \\
\hline & 16 & $\begin{array}{l}\text { Antes de Semana Santa ¿cómo has vivido esta situación de confinamiento en } \\
\text { relación a tu desempeño como docente de EF? }\end{array}$ & Likert, 5 opciones \\
\hline & 17 & $\begin{array}{l}\text { Después de Semana Santa ¿cómo has vivido esta situación de confinamiento en } \\
\text { relación a tu desempeño como docente de EF? }\end{array}$ & Likert, 5 opciones \\
\hline \multirow[t]{4}{*}{$\begin{array}{l}\text { 2. Evaluación y } \\
\text { calificación en EF. }\end{array}$} & 18 & $\begin{array}{l}\text { Durante el confinamiento: ¿has utilizado evaluación formativa (feedback y } \\
\text { comentarios/refuerzos y/o correcciones para alumnos)? }\end{array}$ & Likert, 4 opciones \\
\hline & 19 & $\begin{array}{l}\text { Durante el confinamiento: ¿ha participado el alumnado en la evaluación } \\
\text { (autoevaluación, evaluación entre iguales, evaluación compartida...)? }\end{array}$ & Likert, 4 opciones \\
\hline & 110 & ¿Cómo has calificado el tercer trimestre? & 3 opciones \\
\hline & 111 & ¿Cómo has obtenido la calificación final del curso para cada alumno? & 3 opciones \\
\hline \multirow{2}{*}{$\begin{array}{l}\text { 3. Papel de la } \\
\text { EF durante el } \\
\text { confinamiento. }\end{array}$} & 112 & $\begin{array}{l}\text { ¿Qué papel crees que ha tenido tu asignatura de EF en el día a día de tus } \\
\text { alumnos y sus familias durante el confinamiento? }\end{array}$ & Likert, 4 opciones \\
\hline & 113 & $\begin{array}{l}\text { ¿Qué papel crees que debería tener la EF en el sistema educativo en estas } \\
\text { circunstancias de confinamiento? }\end{array}$ & Likert, 4 opciones \\
\hline
\end{tabular}

\section{Instrumentos}

Se utilizó un único instrumento para la recogida de datos: el cuestionario "Educación Física y Covid-19" (EF\&COVID19). Se trata de un cuestionario anónimo, altamente estructurado. Se elabora y valida ad hoc para el presente estudio, y pretende estudiar cómo la situación de confinamiento ha afectado al trabajo del profesorado de EF en España. El cuestionario ha sido validado previamente mediante dos técnicas: (a) revisión de contenido por jueces externos, llevada a cabo por cuatro reconocidos especialistas, doctores e investigadores de Didáctica de la EF de diferentes universidades españolas; (b) revisión de contenido y forma, llevada a cabo por cinco docentes de EF de Primaria y Secundaria. A lo largo de las dos fases de validación se modificaron algunos ítems, así como alguna de las escalas ordinales utilizadas. El cuestionario está compuesto por 13 ítems cerrados sobre diferentes aspectos de la docencia en la situación de confinamiento. En la mayoría de los ítems se ha utilizado una escala tipo Likert, mientras unos pocos son de opción verbal (ver tabla 5). Se cumplimentaba a través de la aplicación Forms (Office 365).

\section{Dimensiones y Variables}

El cuestionario aborda las siguientes dimensiones y variables, que se relacionan con el objetivo prioritario de nuestro estudio. En la Tabla 5 puede verse la estructura de dimensiones y variables y cada uno de los ítems del cuestionario.

\section{Procedimiento}

Se diseñó el cuestionario entre los investigadores del equipo, los cuales cubrían todas las etapas educativas. Posteriormente se llevaron a cabo los dos procesos consecutivos de validación ya descritos. Una vez fijada la versión definitiva, se difundió a través del correo electrónico y la aplicación WhatsApp mediante un muestreo por "bola de nieve", comenzando por todo el profesorado de EF con el que los investigadores tenían contacto, a la vez que una primera difusión en redes sociales, solicitando enviar el cuestionario al profesorado de EF conocido. Se recogieron datos durante dos meses. Periódicamente se realizaron nuevos avisos a través de las redes sociales (WhatsApp, Twitter, Facebook, etc.) para lograr una mayor difusión del cuestio- 
nario. A finales de julio de 2020 se realizó el análisis de datos. Los datos mostraron que el profesorado participante dedicó una media de unos siete minutos en completar el cuestionario.

\section{Tratamiento de los datos}

Para el tratamiento de los datos se han codificado numéricamente todos los datos obtenidos, y así realizar un análisis estadístico. En los ítems con escala tipo Likert se identificaron los puntos de la escala ordinal con valores numéricos. Por ejemplo, el valor 1 corresponde a la opción Nada; el 2 a Poco; el 3 a Bastante y el 4 a Mucho. En otro caso, por ejemplo: Muy descoordinada (1), Bastante descoordinada (2), Algo descoordinada (3), Bastante coordinada (4), Muy bien coordinada (5). En los resultados pueden comprobarse las diferentes escalas ordinales utilizadas en función de los diferentes ítems. A partir de ahí se procedió a analizar los datos con los programas Excel 2010 y SPSS v22.0, realizando los siguientes análisis estadísticos:

- Descriptivo, para las tres dimensiones. Pruebas descriptivas: medias, desviación típica (DT) y distribución de porcentajes.

- Comparación entre ítems de la dimensión 1 (antes y después de Semana Santa): prueba de T de Student para muestras relacionadas.

\section{Resultados}

Los resultados se organizan en función de las tres dimensiones del cuestionario, tal como se ha explicado en el apartado de metodología.
Diferencias entre las dos etapas temporales (13 de marzo-4 de abril vs. 15 de abril-24 de junio, 2020)

A lo largo de este apartado se analiza el funcionamiento de los centros educativos, el tratamiento de los contenidos de EF y las vivencias de los docentes en dos momentos temporales diferentes, antes y después de las vacaciones de Semana Santa.

Respecto al funcionamiento de los centros educativos, se analiza la capacidad de respuesta y organización del centro educativo antes y después de las vacaciones de Semana Santa según la opinión de los docentes de EF (ver Tabla 6).

En la Tabla 6 se puede comprobar que la distribución de porcentajes es mejor después de Semana Santa. Por ejemplo, el 72,4\% de los encuestados considera que su centro educativo ha tenido un funcionamiento bastante o muy bien coordinado después de Semana Santa, frente a un $52,8 \%$ antes. La media también muestra esta tendencia de mejora, aunque es cierto que son valores altos en ambos casos. La Tabla 7 muestra que las diferencias entre medias son estadísticamente significativas. Por tanto, puede afirmarse que los docentes consideran que ha mejorado el funcionamiento y la organización del centro tras el periodo de vacaciones de Semana Santa.

Respecto a los contenidos de EF trabajados, en las tablas 8 y 9 se puede comprobar en qué grado los docentes han variado sus contenidos y qué contenidos han impartido antes y después de Semana Santa.

Los resultados indican que la mayoría del profesorado ha tenido que realizar cambios importantes en sus programaciones y modificar los contenidos que tenía previsto trabajar. La media ofrece un valor muy elevado (3.11, en una escala 1-4).

Tabla 6. Funcionamiento del centro escolar antes y después de las vacaciones de Semana Santa.

\begin{tabular}{|c|c|c|c|c|}
\hline & \multicolumn{2}{|c|}{ Antes } & \multicolumn{2}{|c|}{ Después } \\
\hline & Frecuencia & Porcentaje & Frecuencia & Porcentaje \\
\hline Muy descoordinada (1) & 44 & 8.4 & 19 & 3.6 \\
\hline Bastante descoordinada (2) & 74 & 14.1 & 40 & 7.6 \\
\hline Algo descoordinada (3) & 130 & 24.7 & 86 & 16.3 \\
\hline Bastante coordinada (4) & 211 & 40.1 & 234 & 44.5 \\
\hline Muy bien coordinada (5) & 67 & 12.7 & 147 & 27.9 \\
\hline Total & 526 & 100 & 526 & 100 \\
\hline Media & \multicolumn{2}{|c|}{3.35} & \multicolumn{2}{|c|}{3.86} \\
\hline DT (Desviación Típica) & \multicolumn{2}{|c|}{1.12} & \multicolumn{2}{|c|}{1.03} \\
\hline
\end{tabular}

Tabla 7. Prueba T. Diferencia de medias entre el funcionamiento del centro escolar antes y después de las vacaciones de Semana Santa.

\begin{tabular}{llll}
\hline \multirow{2}{*}{$\begin{array}{l}\text { ¿ómo consideras que fue la respuesta del centro/equipo directivo ante la situación creada } \\
\text { con el confinamiento de escolares (a nivel de coordinación entre profesores, etc.)? }\end{array}$} & \multicolumn{2}{c}{ t } & gl Sig. (bilateral) \\
\cline { 2 - 3 } & 15.018 & 525
\end{tabular}


Tabla 8. Variación de los contenidos programados por la pandemia.

\begin{tabular}{|c|c|c|}
\hline & Sujetos & $\%$ \\
\hline Nada (1) & 11 & 2.1 \\
\hline Poco (2) & 73 & 13.9 \\
\hline Bastante (3) & 289 & 54.9 \\
\hline Mucho (4) & 153 & 29.1 \\
\hline Total & 526 & 100 \\
\hline Media & \multicolumn{2}{|c|}{3.11} \\
\hline DT & \multicolumn{2}{|c|}{0.71} \\
\hline
\end{tabular}

Tabla 9. Contenidos de EF trabajados, organizados por fases. la primera fase, con un crecimiento muy notable en la segunda fase. En cuanto a la comparativa entre los dos intervalos temporales, las diferencias más llamativas son: (a) el bloque 2 se trabaja todavía menos que en la fase anterior, reduciéndose a valores muy bajos; (b) los bloques 4 y 5 sufren un fuerte incremento.

Respecto a cómo han vivido los docentes esta situación de enseñanza on-line durante el periodo de confinamiento, en las Tablas 10 y 11 puede comprobarse que los docentes la han vivido de manera diferente antes y después del periodo vacacional, siendo estas diferencias estadísticamente significativas.

\begin{tabular}{|c|c|c|c|c|}
\hline \multirow[t]{2}{*}{ Bloques de contenidos en EF en España } & \multicolumn{2}{|c|}{ Antes } & \multicolumn{2}{|c|}{ Después } \\
\hline & Frecuencia & Porcentaje & Frecuencia & Porcentaje \\
\hline 1. Acciones motrices individuales en entorno estable. & 400 & 76.0 & 410 & 77.9 \\
\hline 2. Acciones motrices en oposición interindividual. & 65 & 12.4 & 46 & 8.7 \\
\hline 3. Acciones motrices de cooperación y de colaboración-oposición. & 110 & 20.9 & 113 & 21.5 \\
\hline 4. Acciones motrices de adaptación a un entorno inestable (medio natural). & 108 & 20.5 & 268 & 51.0 \\
\hline 5. Acciones motrices de índole artístico y expresivo. & 240 & 45.6 & 354 & 67.3 \\
\hline
\end{tabular}

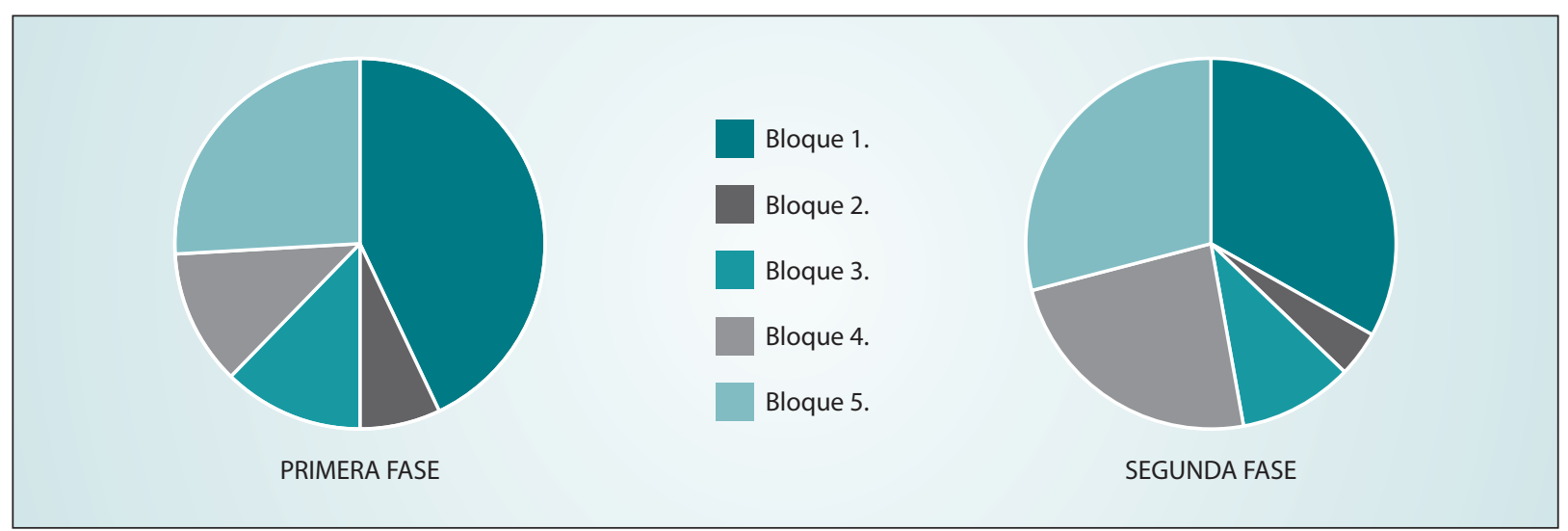

Figura 1. Distribución de contenidos trabajados en la primera fase del confinamiento.

La Tabla 9 y la Figura 1 permiten analizar qué contenidos son los que más ha trabajado el profesorado de EF durante el confinamiento, así como las diferencias entre los dos intervalos temporales. Para ello, se han utilizado los bloques de contenidos correspondientes al área de EF del currículum oficial del Ministerio para Educación Primaria (RD 126/2014) y Educación Secundaria (RD 1105/2014).

Los datos muestran un claro predominio del bloque 1 en una primera fase. También muestran un trabajo muy bajo del bloque 2 y bajo de los bloques 3 y 4 . El bloque 4 se trabaja muy poco en la fase de confinamiento obligatorio, pero sufre un incremento considerable en la segunda fase, cuando ya se permite realizar actividad física al aire libre en horario reducido. El bloque 5 es trabajado por casi la mitad del profesorado en
Antes de Semana Santa la mayoría de los docentes (75\%) han sentido en su desempeño docente bastante o mucha inseguridad e incertidumbre, frente a un 52\% después de Semana Santa.

\section{Evaluación y calificación}

Las dos primeras preguntas hacen referencia a la utilización regular de la evaluación formativa y al fomento de la participación del alumnado en los procesos de evaluación (Tablas 12 y 13). Las preguntas tres y cuatro se centran en los criterios que el profesorado ha tenido en cuenta para fijar la calificación de su alumnado al finalizar el tercer trimestre y al final del curso (Tablas 14 y 15). En la Tabla 12 se puede comprobar que los docentes de EF han utilizado evaluación 
Tabla 10. Cómo han vivido los docentes esta situación antes y después de Semana Santa.

\begin{tabular}{|c|c|c|c|c|}
\hline & \multicolumn{2}{|c|}{ Antes } & \multicolumn{2}{|c|}{ Después } \\
\hline & Frecuencia & Porcentaje & Frecuencia & Porcentaje \\
\hline Con nada de inseguridad e incertidumbre (1) & 19 & 3.6 & 55 & 10.5 \\
\hline Con poca inseguridad e incertidumbre (2) & 113 & 21.5 & 200 & 38.0 \\
\hline Con bastante inseguridad e incertidumbre (3) & 258 & 49.0 & 203 & 38.6 \\
\hline Con mucha inseguridad e incertidumbre (4) & 136 & 25.9 & 68 & 12.9 \\
\hline Total & 529 & 100 & 526 & 100.0 \\
\hline Media & \multicolumn{2}{|c|}{2.97} & \multicolumn{2}{|c|}{2.54} \\
\hline DT & \multicolumn{2}{|c|}{0.77} & \multicolumn{2}{|c|}{0.85} \\
\hline
\end{tabular}

Tabla 11. Prueba T. Diferencia de medias entre antes y después de Semana Santa.

\begin{tabular}{lccc}
\hline ¿Cómo has vivido esta & t & gl & Sig. (bilateral) \\
\cline { 2 - 4 } $\begin{array}{l}\text { situación de confinamiento } \\
\text { en relación a tu desempeño } \\
\text { como docente de EF? }\end{array}$ & 11.649 & 525 & 0.000 \\
\hline
\end{tabular}

Tabla 12. Utilización de la evaluación formativa durante el confinamiento.

\begin{tabular}{lcc}
\hline & Frecuencia & Porcentaje \\
\hline Nada (1) & 16 & 3.0 \\
Poco (2) & 104 & 19.8 \\
Bastante (3) & 206 & 39.2 \\
Mucho (4) & 200 & 38.0 \\
Total & 526 & 100.0 \\
Media & & 3.12 \\
DT & & 0.83 \\
\hline
\end{tabular}

formativa (conocimiento de resultados y comentarios/ refuerzos y/o correcciones para alumnos) durante el confinamiento en un alto grado (3.12 de media en una escala 1-4) y en la mayoría de los casos.

Los resultados de la Tabla 13 muestran que la participación del alumnado en los procesos de evaluación (autoevaluación, evaluación entre iguales, evaluación compartida...) parece haber sido poco utilizada por los docentes de EF (1.95 de media, escala 1-4), aunque aparecen porcentajes interesantes de docentes de EF que han promovido bastante o mucho la participación del alumnado en evaluación durante estos meses de confinamiento.

Tabla 13. Participación de los estudiantes en la evaluación en EF.

\begin{tabular}{|c|c|c|}
\hline & Frecuencia & Porcentaje \\
\hline Nada (1) & 176 & 33.5 \\
\hline Poco (2) & 226 & 43.0 \\
\hline Bastante (3) & 96 & 18.3 \\
\hline Mucho (4) & 28 & 5.3 \\
\hline Total & 526 & 100.0 \\
\hline Media & \multicolumn{2}{|c|}{1.95} \\
\hline DT & \multicolumn{2}{|c|}{0.85} \\
\hline
\end{tabular}

Respecto a la pregunta sobre cómo los docentes de EF han calificado lo realizado durante el confinamiento (tercer trimestre), la Tabla 14 muestra que un elevado porcentaje del profesorado parece haber utilizado procesos de evaluación continua (68.8\%), basados en las tareas o actividades realizadas por el alumnado, sin necesidad de realizar pruebas ni exámenes puntuales, opción que solo utiliza un $2.7 \%$. En cambio, casi un tercio del profesorado dice combinar ambas opciones (evaluación continua y alguna prueba puntual).

Tabla 14. Cómo se califica el tercer trimestre.

\begin{tabular}{lcc}
\hline & Frecuencia & Porcentaje \\
\hline $\begin{array}{l}\text { Puntual (exámenes, } \\
\text { cuestionarios, pruebas) }\end{array}$ & 14 & 2.7 \\
$\begin{array}{l}\text { Evaluación continua (tareas/ } \\
\text { actividades realizadas a lo largo } \\
\text { del trimestre) }\end{array}$ & 362 & 68.8 \\
$\begin{array}{l}\text { Combinación de ambas } \\
\text { Total }\end{array}$ & 150 & 28.5 \\
\hline
\end{tabular}

Los resultados de la Tabla 15 muestran que la mayoría del profesorado de EF ha optado por solo tener en cuenta los dos primeros trimestres del curso escolar para obtener la calificación final del curso de cada alumno, frente a los que optan por dar a los tres trimestres el mismo peso o tener en cuenta el tercer trimestre solo en los estudiantes que han trabajado bien durante el confinamiento y, por tanto, les sirve para mejorar la calificación de los trimestres anteriores.

Tabla 15. Cómo se califica el curso completo.

\begin{tabular}{lcc}
\hline & Frecuencia & Porcentaje \\
\hline $\begin{array}{l}\text { Los tres trimestres han tenido el } \\
\text { mismo peso }\end{array}$ & 30 & 5.7 \\
$\begin{array}{l}\text { Solo he tenido en cuenta primer } \\
\text { y segundo trimestre para todos }\end{array}$ & 444 & 84.4 \\
$\begin{array}{l}\text { He tenido en cuenta solo primer } \\
\text { y segundo trimestre en alumnos } \\
\text { desconectados y también el } \\
\text { tercer trimestre si sube nota }\end{array}$ & 52 & 9.9 \\
\begin{tabular}{l} 
Total \\
\hline
\end{tabular} & 526 & 100.0 \\
\hline
\end{tabular}


Sensaciones vividas por el profesorado y papel de la EF durante el confinamiento

Tal y como muestra la Tabla 16, más de la mitad de los docentes de EF (55,3\%) considera que su asignatura ha tenido un papel importante o muy importante durante el confinamiento.

Tabla 16. Papel de la asignatura de Educación Física durante el confinamiento.

\begin{tabular}{lcc}
\hline & Frecuencia & Porcentaje \\
\hline Inexistente (1) & 4 & 0.8 \\
Escaso (2) & 231 & 4.9 \\
Importante (3) & 213 & 40.5 \\
Muy importante (4) & 78 & 14.8 \\
Total & 526 & 100.0 \\
Media & \multicolumn{2}{c}{2.79} \\
DT & \multicolumn{2}{c}{0.73} \\
\hline
\end{tabular}

En la Tabla 17 se observa que la mayoría de los docentes (97.3\%) cree que su asignatura debería tener un papel importante o muy importante en el día a día de sus estudiantes en esta situación de confinamiento.

Tabla 17. Papel que debería tener la asignatura de Educación Física durante el confinamiento.

\begin{tabular}{lcc}
\hline & Frecuencia & Porcentaje \\
\hline Inexistente (1) & 2 & 0.4 \\
Escaso (2) & 12 & 2.3 \\
Importante (3) & 251 & 47.7 \\
Muy importante (4) & 261 & 49.6 \\
Total & 526 & 100.0 \\
Media & & 3.47 \\
DT & \multicolumn{3}{c}{0.56} \\
\hline
\end{tabular}

\section{Discusión}

En primer lugar, se ha intentado comprobar la hipótesis de que existen diferencias entre los dos periodos temporales estudiados. Los resultados parecen indicar que el cierre inesperado de centros escolares generó una notable confusión e incertidumbre entre el profesorado de EF. Tanto los centros como los profesores tuvieron que improvisar cómo trabajar ante la nueva situación generada: confinamiento de toda la población en sus casas y sistemas de educación a distancia. El profesorado de EF tuvo que reorganizar repentinamente una asignatura que es claramente presencial, práctica, social y con espacios y materiales específicos, en una situación radicalmente diferente: los estudian- tes confinados en sus casas, en muchos casos sin espacio suficiente, sin materiales y sin poder interactuar con otros compañeros (salvo familias con hermanos de edades cercanas). Los datos muestran diferencias considerables en todas las variables estudiadas.

En lo referido a la organización de los centros educativos y coordinación entre el profesorado, hay diferencias estadísticamente significativas entre el antes y el después del periodo de vacaciones. Estos resultados son similares a los encontrados en el estudio de Fuentes et al. (2020), donde se afirma que los docentes redujeron sus niveles de agobio, inseguridad e incertidumbre después del periodo de Semana Santa y que los centros educativos dieron una gran responsabilidad al profesor tutor de cada curso. Esta mejoría observada hace pensar que el periodo de vacaciones fue un momento idóneo para reflexionar y reorganizar el funcionamiento de los centros escolares, así como las intervenciones de los diferentes docentes. Además, los intercambios de recursos y experiencias entre docentes de EF (Martínez-Egea, 2020), pudieron contribuir a una reorganización de las programaciones didácticas y una mayor coordinación entre los docentes de un mismo centro.

Los resultados también muestran que la mayoría del profesorado de EF ha tenido que realizar cambios muy importantes en sus programaciones y modificar los contenidos que tenía previsto trabajar en estas fechas. En los contenidos trabajados se observan importantes diferencias entre los dos periodos temporales. En el primer periodo predominan las acciones motrices individuales en entorno estable (bloque 1), al igual que en el estudio de Baena-Morales et al. (2021). En cambio, en la segunda fase aparecen otros dos bloques que son trabajados por un porcentaje elevado del profesorado: (1) acciones motrices de índole artístico y expresivo (bloque 5) y (2) acciones motrices de adaptación a un entorno inestable (bloque 4). Es curioso que un bloque de contenidos que tradicionalmente suele ser poco trabajado (la "expresión corporal"), haya tenido un crecimiento tan considerable en esta fase de confinamiento. Una posible explicación es que este tipo de actividades pueden realizarse en espacios reducidos, y además permiten la grabación de evidencias con el smartphone, incluso la realización de trabajos en grupo (grabaciones individuales que posteriormente se fusionan en un único video) que son enviados al docente. En este sentido, es clave la formación digital del profesorado que permita una mayor motivación y participación del alumnado en el proceso educativo a distancia (Calderón et al., 2020).

El incremento notable de las acciones motrices en entorno inestable puede deberse al proceso de des- 
confinamiento. A partir del mes de mayo se permitió salir a realizar actividades físicas al aire libre una vez al día. Los datos parecen mostrar que el profesorado de EF aprovechó la situación para encargar a su alumnado tareas y actividades a realizar al aire libre como parte del trabajo de la asignatura.

En contraposición, hay dos bloques de contenidos muy poco utilizados: las acciones motrices en oposición interindividual (bloque 2) y las acciones motrices de cooperación y de colaboración-oposición (bloque $3)$. Sus bajos resultados se pueden explicar porque se trata de actividades físicas que suelen requerir otras personas para poder realizarse y, en muchas ocasiones, espacios amplios y materiales específicos. La primera condición solo la cumplen los estudiantes que conviven con otros hermanos de edades cercanas, o bien requieren la implicación de la familia. Sin embargo, no en todas las familias se disponía de tiempo suficiente (teletrabajo, trabajadores esenciales fuera del hogar, otras ocupaciones familiares...) para realizar este tipo de actividades colectivas (Fuentes et al., 2020). Otros inconvenientes, como la falta de medios digitales en la familia o el estrés que ha generado la enseñanza a distancia en las familias, pueden encontrarse también en García et al. (2020) en España, así como en Australia (Brown et al., 2020). Martínez-Egea (2020) también encuentra diferencias entre los contenidos trabajados en EF a lo largo del confinamiento.

Los resultados muestran diferencias importantes y estadísticamente significativas entre las sensaciones $\mathrm{y}$ vivencias de los docentes de EF en los dos periodos estudiados. En el primer periodo, la mayoría de los docentes vivieron la situación con inseguridad e incertidumbre. Tras el periodo vacacional estas sensaciones no desaparecieron, pero fueron disminuyendo considerablemente. Estos resultados coinciden con el estudio de Fuentes et al. (2020). Parece que los docentes se fueron adaptando paulatinamente al confinamiento y a la docencia on-line. Otros autores coinciden en que los docentes de EF vivieron una situación de incertidumbre que fue disminuyendo con el tiempo (Martínez-Egea, 2020).

Respecto a los procesos de evaluación y calificación llevados a cabo, los datos muestran que la mayoría de los docentes de EF afirman haber desarrollado procesos de evaluación formativa con sus estudiantes durante el confinamiento (feedback y comentarios/refuerzos y/o correcciones para estudiantes). Creemos que hay varias explicaciones de estos resultados. Por un lado, los procesos de evaluación formativa y feedback se pueden haber visto facilitados por la variedad de herramientas informáticas con las que se ha mantenido la comunicación con estudiantes y familias durante toda la fase de confinamiento: plataformas virtuales (Baena-Morales et al., 2021), correo electrónico, chat, videoconferencias, etc. (Fuentes et al., 2020). Por otra parte, es un tipo de evaluación que resulta también útil para el profesorado y el mejor funcionamiento de la asignatura. El primer aspecto puede ser explicado porque la imposibilidad de aportar un feedback inmediato y presencial ha hecho que los docentes generen procesos de evaluación formativa a través de diferentes herramientas, entendiendo la evaluación como un proceso de diálogo y mejora profesor-alumno (Santos-Guerra, 1993). Dado que la enseñanza a distancia dificulta el seguimiento continuo del estudiante, muchos docentes han basado su metodología de trabajo en el envío de tareas periódicas (semanales, fundamentalmente) y la revisión de cada una de ellas y su correspondiente feedback, generando procesos de evaluación formativa y continua. Una de las características más básicas de este modelo de "evaluación formativa y compartida" es que no está centrado solamente en mejorar los procesos de aprendizaje del alumnado, sino que debe ser útil también para que el profesorado aprenda y mejore su calidad docente, y para poder evaluar y mejorar sobre la marcha los procesos de enseñanza-aprendizaje que tienen lugar en la asignatura (Herrero-González et al., 2020; López-Pastor, 2006). Estos procesos formativos en las tres dimensiones han ocurrido claramente durante todo este proceso de confinamiento, donde tanto estudiantes como docentes han tenido que aprender día a día ante una situación inesperada y que inicialmente ha superado a todo el mundo. Son precisamente estos procesos de evaluación formativa y compartida los que han permitido a docentes, estudiantes y familias aprender a resolver los problemas que se han ido planteando, para poder mantener los mejores procesos de aprendizaje posibles en una situación tan difícil y novedosa.

En cambio, los datos muestran que la participación del alumnado en los procesos de evaluación (autoevaluación, evaluación entre iguales, evaluación compartida...) es más bien escasa y parece haber sido utilizada por un porcentaje reducido del profesorado de EF. Quizás, esa baja participación del alumnado en la evaluación pueda deberse a las limitaciones propias del uso de las TIC o a las dificultades de conectar con todos los estudiantes. Existen numerosas evidencias de que es posible fomentar esta participación en la evaluación y que tiene considerables ventajas en el aprendizaje y la autorregulación del mismo (Fernández del Río, 2020; López-Pastor, 2006; López-Pastor et al., 2013; LorenteCatalán, 2008; MacPhail y Halbert, 2010).

En lo que respecta a la calificación del tercer trimestre, los datos muestran que un elevado porcentaje de 
los docentes de EF afirma haber utilizado procesos de evaluación continua, aunque casi un tercio de los docentes de EF combina ambas opciones (evaluación continua y alguna prueba puntual). La situación de pandemia parece haber generado un avance educativo en el sentido de que más docentes de EF estén realizando una evaluación integrada; esto es, que no necesite realizar pruebas puntuales para obtener la calificación del alumno, y que los procesos de aprendizaje y evaluación continuos desarrollados durante el trimestre ofrecen la suficiente información como para fijar la calificación (AEISEP, 2020; López-Pastor et al., 2013). Pero también puede tratarse de un hecho puntual y artificial, debido a la gran dificultad de realizar pruebas y exámenes de carácter motriz en una situación de enseñanza on-line como la que se ha vivido. En esta situación es mucho más sencillo evaluar a través de retos motrices o proyectos que los estudiantes han de realizar por su cuenta, grabar en vídeo y enviar al docente, que la realización de pruebas en tiempo real.

Respecto a la calificación final del curso, resulta extraño que solo el $10 \%$ del profesorado se haya ceñido de forma estricta a lo que marca la legislación educativa para esta situación de confinamiento: basarse en el trabajo realizado en primer y segundo trimestre; la nota final no puede ser inferior a la obtenida en los dos primeros trimestres; el tercer trimestre puede servir para subir algo la nota a los estudiantes que han desarrollado correctamente los procesos de aprendizaje (Orden EFP/365/2020 de 22 de abril). ¿Cuál es el motivo por el que la mayoría del profesorado opta por tener en cuenta solo los dos primeros trimestres para obtener la calificación final del curso de cada estudiante? Se han encontrado tres posibles explicaciones: (a) haber interpretado o entendido mal las instrucciones de la legislación educativa que lo regulaba; (b) acuerdos del centro, que han optado por un sistema más garantista para los estudiantes que se han quedado desconectados por la situación de confinamiento o que se han visto afectados directamente por la Covid-19 (ellos o sus familiares); (c) las calificaciones del primer y segundo trimestre no se han visto alteradas al tener en cuenta el tercero solo de manera positiva, por lo que el profesorado puede no haber entendido bien las diferentes opciones de la pregunta.

Por último, se quiso comprobar qué opinan los docentes de EF del papel que ha tenido y el que debería haber tenido su asignatura durante este periodo. Este estudio revela que algo más de la mitad de los docentes de EF cree que la asignatura ha tenido un papel importante o muy importante durante una situación de confinamiento como la vivida. No obstante, la amplia mayoría cree que el papel de la EF debería ser impor- tante o muy importante en este tipo de situaciones, en las que es fundamental evitar el sedentarismo y mantener un nivel adecuado de actividad física tanto en la población general como entre los escolares (Shaidi et al., 2020). La asignatura de EF puede contribuir al mantenimiento y mejora de la salud física y mental en esta situación excepcional. Teniendo en cuenta estos datos, parece necesario que la comunidad educativa en su conjunto (administraciones, equipos directivos de los centros, padres, profesores y alumnos) tome conciencia de la importancia de la EF, especialmente en situaciones de confinamiento como la vivida.

\section{Conclusiones}

Los resultados muestran que existen diferencias estadísticamente significativas entre los dos periodos temporales (antes y después de Semana Santa) en todas las variables estudiadas: (a) organización de los centros y coordinación entre el profesorado; (b) contenidos de EF trabajados; (c) las sensaciones y vivencias de los docentes de EF ante la situación vivida. Por tanto, los datos indican que el cierre brusco e inesperado de centros escolares generó una notable confusión e incertidumbre entre el profesorado de EF, que tuvo que improvisar inicialmente para aprender a trabajar ante la nueva situación de confinamiento y educación a distancia. Ahora bien, el periodo de vacaciones de Semana Santa parece haber sido fundamental para reflexionar y reorganizar el funcionamiento de los centros escolares y de la reorganización de la asignatura de EF. En este sentido, la mayoría de los profesores afirma haber realizado cambios muy importantes en las programaciones que tenía preparadas. La situación de confinamiento y la posterior desescalada han generado cambios importantes en los contenidos trabajados en EF. Por último, los resultados muestran diferencias importantes y estadísticamente significativas entre las sensaciones y vivencias de los docentes de EF en los dos periodos señalados.

En segundo lugar, la mayoría del profesorado de EF parece haber utilizado predominantemente sistemas de evaluación formativa durante todo este proceso de enseñanza a distancia, tanto para los procesos de aprendizaje del alumnado como para los propios procesos de mejora de la capacidad y metodología docente. En cambio, los datos muestran que la participación del alumnado en los procesos de evaluación es poco habitual. En lo que respecta a la calificación del tercer trimestre, se observa que un elevado porcentaje de los docentes de EF afirma haber utilizado procesos de evaluación continua, aunque también existe casi un tercio 
de ellos que combina la evaluación continua con alguna prueba puntual. Respecto a la calificación final del curso, resulta curioso comprobar que la mayoría del profesorado dice haber tenido en cuenta solo la primera y segunda evaluación.

En tercer lugar, los resultados muestran que algo más de la mitad del profesorado de EF cree que su asignatura ha tenido un papel importante o muy importante durante una situación de confinamiento como la vivida, mientras que la mayoría considera que el papel de la EF debería ser importante o muy importante en este tipo de situaciones, en las que es fundamental evitar el sedentarismo y mantener un nivel adecuado de actividad física.

La principal limitación del estudio es que la muestra no ha sido seleccionada aleatoriamente. A pesar de ello, se han recogido cuestionarios de docentes de EF de todas las comunidades autónomas españolas y es una muestra lo suficientemente grande. Otra limitación puede estar relacionada con que no se han investigado todas las temáticas posibles, sino solo las señaladas en los objetivos y que se ha aplicado en el mes de junio y julio, por lo que la saturación y sobrecarga del profesorado puede que haya influido negativamente en lograr una muestra mayor.

Este estudio aporta una interesante radiografía de cómo el profesorado de EF ha vivido la situación repentina de confinamiento y educación a distancia generada por la pandemia Covid-19 en España entre los meses de marzo y junio de 2020. Estos resultados pueden ser de interés para el profesorado de EF en ejercicio, tanto a la hora de aprender de lo vivido como, sobre todo, a la hora de plantearse cómo enfocar el curso $2020 / 21$, en que la situación generada por esta pandemia va a seguir afectando considerablemente nuestra asignatura, tanto si tenemos que trabajar en educación presencial como si toca volver a situaciones de educación a distancia de forma puntual. Por último, puede ser de utilidad a los investigadores especializados en didáctica de la EF sobre cómo adaptar la enseñanza a las nuevas situaciones que se están generando.

Aunque están comenzando a publicarse algunos trabajos sobre la situación del área de EF durante la pandemia Covid-19, creemos que es importante continuar profundizando en lo que ha ocurrido en este complejo periodo del curso 2019/20 y lo que está ocurriendo en el curso actual (2020/21), especialmente de cara a preparar protocolos y estrategias específicas por si vuelven a darse situaciones similares en los próximos años (Grupo asesores de enseñanza de la Educación Física, 2020). Por ejemplo, puede ser interesante investigar qué aplicaciones y herramientas TIC han sido las más utilizadas por el profesorado de EF durante el periodo de confinamiento. También parece importante realizar estudios longitudinales que abarquen todo el periodo de la Covid-19 y reflejen los cambios tan importantes y rápidos que se están llevando a cabo en esta situación tan excepcional. 


\section{BIBLIOGRAFÍA}

AIESEP (2020). Declaración oficial AIESEP sobre Evaluación en Educación Física. AIESEP. https://aiesep.org/scientificmeetings/position-statements//

Baena-Morales, S., López-Morales, J., \& García-Taibo, O. (2021). La intervención docente en educación física durante el periodo de cuarentena por Covid-19. Retos, 39, 388-395. https://doi.org/10.47197/ retos.v0i39.80089.

Blázquez, D. (2017). Cómo evaluar bien en EF. El enfoque de la evaluación formativa. Inde: Barcelona.

Brown, N., Riele, K., Shelley, B., \& Woodroffe, J. (2020). Learning at home during Covid-19: Effects on vulnerable young Australians. Independent Rapid Response Report. Hobart: University of Tasmania. doi:10.1080 /00131911.2020.1728232

Calderón, A., Meroño, L., \& MacPhail, A. (2020). A student-centred digital technology approach: The relationship between intrinsic motivation, learning climate and academic achievement of physical education pre-service teachers. European Physical Education Review, 26(1), 241-262. doi:10.1177/1356336X19850852

Fernández del Río, J. (2020). Apuntes metodológicos para una educación física Post-covid-19. EmásF, Revista Digital de Educación Física, 11(66), 67-75. https://www.researchgate.net/publication/344130739

Fuentes, T., Jiménez, B., \& López-Pastor, V. M. et al. (2020). Educación Física en tiempos de coronavirus y confinamiento: análisis desde la experiencia. EmásF, Revista Digital de Educación Física, 11(65), 151181. http://emasf2.webcindario.com/EmasF_65.pdf

García, N.; Rivero, M. L., \& Ricis, J. (2020). Brecha digital en tiempo del Covid-19. Revista Educativa Hekademos, 28, 76-85. https://www.hekademos.com/index.php/hekademos/article/view/9

Grupo asesores de enseñanza de la Educación Física (2020). Recomendaciones docentes para una educación física escolar segura y responsable ante la "nueva normalidad". Minimización de riesgos de contagio de la Covid-19 en las clases de EF para el curso 2020-2021. Revista española de educación física y deportes, 429, 81-93.

Herrero-González, D., López-Pastor, V.M., \& Manrique-Arribas, J.C. (2020). La Evaluación Formativa y Compartida en contextos de Aprendizaje Cooperativo en Educación Física en Primaria. Cultura, Ciencia y Deporte, 15(44), 213-222. doi:10.12800/ccd.v15i44.1463.

Julián, J. A., Abarca-Sos, A, Zaragoza, J., \& Aibar, A. (2016). Análisis crítico de la propuesta del currículum básico de la LOMCE para la asignatura de Educación Física. Acciones derivadas en la Comunidad Autónoma de Aragón y propuestas de futuro. Retos de la Actividad Física y el Deporte, 29, 173-181. doi:10.47197/retos.v0i29.40968

Larranz, A. (2004). Los dominios de acción motriz como base de los diseños curriculares en educación física: el caso de la Comunidad de Aragón en educación primaria. En Lagardera, F. y Lavega, P. (Ed.) La ciencia de la acción motriz. (203-226). Lleida: Universitat de Lleida.

López-Pastor V. M., \& Pérez-Pueyo, A. (Coords.) (2017). Evaluación formativa y compartida en Educación: experiencias de éxito en todas las etapas educativas. León: Universidad de León.

López-Pastor, V. M. (Coord.) (2006). La Evaluación en Educación Física: Revisión de los modelos tradicionales y planteamiento de una alternativa: La evaluación formativa y compartida. Buenos Aires: Miño y Dávila.
López-Pastor, V. M., et al (2016). Veinte años de formación permanente del profesorado, investigación-acción y programación por dominios de acción motriz. RETOS. Nuevas Tendencias en Educación Física, Deporte y Recreación, (29),270-279.

López-Pastor, V. M., Kirk, D., Lorente-Catalán, E., MacPhail, A., \& Macdonald, D. (2013). Alternative Assessment in Physical Education: A Review of International Literature. Sport, Education \& Society, 18(1), 57-76. doi:10.1080/13573322.2012.713860.

Lorente-Catalán, E. (2008). Estimular la responsabilidad y la iniciativa: autogestión en educación física. Apunts. Educació física i esports, 92(2), 2634. https://www.raco.cat/index.php/ApuntsEFE/article/view/299952

MacPhail, A., \& Halbert, J. (2010) 'We had to do intelligent thinking during recent PE': students' and teachers' experiences of assessment for learning in post-primary physical education. Assessment in Education: Principles, Policy \& Practice, 17(1), 23-39. doi:10.1080/09695940903565412

Martínez-Egea, B. (2020). Educación Física post Covid-19 ¿Evolución o deriva?, Sociología del Deporte, 1(1), 45-48. doi:10.46661/socioldeporte. 5010

Martínez-Hita, F.J. (2020). Propuestas activas en Educación Física durante el confinamiento por el Covid-19. Lecturas: Educación Física y Deportes, 25(266), 2-13. doi:10.46642/efd.v25i266.2178

ORDEN EFP/365/2020, de 22 de abril, por la que se establecen el marco y las directrices de actuación para el tercer trimestre del curso 2019-2020 y el inicio del curso 2020-2021, ante la situación de crisis ocasionada por el Covid-19 (BOE Núm. 114, de 24 de abril, 1-15). https://www.boe.es/ boe/dias/2020/04/24/pdfs/BOE-A-2020-4609.pdf

Real Decreto 1105/2014, de 26 de diciembre, por el que se establece el currículo básico de la Educación Secundaria Obligatoria y del Bachillerato. Ministerio de Educación, Cultura y Deporte (BOE,3, de 3 de enero de 2015). https://www.boe.es/eli/es/rd/2014/12/26/1105/con

Real Decreto 126/2014, de 28 de febrero, por el que se establece el currículo básico de la Educación Primaria. Ministerio de Educación, Cultura y Deporte (BOE, 52, de 1 de marzo de 2014). https://www.boe.es/buscar/ pdf/2014/BOE-A-2014-2222-consolidado.pdf

Real Decreto 463/2020, de 14 de marzo, por el que se declara el estado de alarma para la gestión de la situación de crisis sanitaria ocasionada por el Covid-19 (BOE Núm. 67, de 14 de marzo). https://www.boe.es/ buscar/act.php?id=BOE-A-2020-3692

Sanmartín, N. (2007). 10 ideas clave: evaluar para aprender. Grao: Barcelona. Santos-Guerra, M. Á. (1993). La evaluación, un proceso de diálogo, comprensión y mejora. Aljibe: Archidona.

Shahidi, S.H., Stewart, J., \& Hassani, F. (2020), Physical activity during Covid-19 quarantine. Acta Paediatrica, 109(10), 2147-2148. doi:10.1111/apa.15420

Silva-Filho, E., Teixeira, A.L., Xavier, J.R., Braz, D., Barbosa, R.A., \& Albuquerque, J.A. (2020). Physical education role during coronavirus disease 2019 (Covid-19) pandemic Physical education and Covid-19. Motriz: Revista de Educação Física, 26(2). doi:10.1590/ s1980-6574202000020086

World Health Organization (WHO) (2010). World Health Organization Global Recommendations on Physical Activity for Health. Geneva, Switzerland. 